www.jmscr.igmpublication.org

Impact Factor 5.84

Index Copernicus Value: 83.27

ISSN (e)-2347-176x ISSN (p) 2455-0450

crossref DOI: _https://dx.doi.org/10.18535/jmscr/v5i5.81

\author{
Journal Of Medical Science And Clinical Research \\ IGM Publication \\ An Official Publication of IGM Publication
}

\title{
Propofol - A Gallery of Different Formulations and Methods to Alleviate Pain on Injection
}

\author{
Author \\ Vaijayanti Nitin Gadre
}

Department of Anaesthesiology, B. J. Government Medical College, Pune (Maharashtra state), India Mobile number-9075618317, 9921192739 Email: vaijayantigadre@ hotmail.com

\begin{abstract}
Propofol is a popular intravenous (IV) anaesthetic agent both for induction and maintenance. The initial formulations were potent lipid emulsions; efforts to overcome their drawbacks have altered propofol preparation over the decades. Propofol soybean emulsion has been largely acceptable because it is composed of substances endogenous to the body. Pain on injection, allergic reactions, contamination and hyperlipidaemia are the risk factors that occur frequently. Pain on injection is still an unsolved clinical problem during anaesthesia. Various pre-treatment and induction techniques that have been investigated to minimize the incidence of pain have been discussed here and will find the interest of the readers.
\end{abstract}

Keywords-Propofol, IV Injection, Pain on Injection.

\section{INTRODUCTION}

Propofol (Diprivan-ICI 35868) is a commonly used IV induction agent. Primarily a hypnotic, this alkylphenol is formulated in a lipid emulsion form. The duration of hypnosis in the induction dose 1 to $2 \mathrm{mg} / \mathrm{kg}$, is 5 to 8 minutes, followed by a rapid clear headed recovery. There is depression of ventilation in therapeutic dose depending upon the speed of injection and concomitant premedication. ${ }^{[1]}$ Hypotension due to decrease in cardiac output and vasodilatation mediated by endothelial nitric oxide synthase activation simultaneously resets the baroreflex rather than inhibiting it. ${ }^{[2]}$ The common side-effect during induction is pain on injection; an undesirable property due to lipid based formulation. Additional effects like inadvertent bacterial contamination, ${ }^{[3]}$ serious allergic reactions ${ }^{[4,5]}$ and Propofol infusion syndrome ${ }^{[6]}$ are a function of the lipid base.

\section{DISCUSSION}

\subsection{Different formulations of propofol derived for the control of injection pain-}

The commercial preparation has undergone modification over years. The first clinical trial in 1977 was conducted using 2, 6-diisopropylphenol 1\% formulated in Cremophor EL. Propofol being highly lipophilic, a vehicle, which could keep the active ingredient evenly dispersed in an aqueous solution, was required. Cremophor EL used as a vehicle, was non-sedative, non-anaesthetic, and non-toxic and allowed propofol to reside in the hydrophobic core of its micelle. First human trial of propofol $(2 \%)$ contained Cremophor EL (16\% polyethylated castor oil) and $8 \%$ ethanol. ${ }^{[7]}$

Subsequently, $1 \%$ formulation was adopted to reduce the pain of injection and ethanol was eliminated. However, the possibility of hypersensitivity reaction was not ruled out. ${ }^{[8]}$ 
When used for anaesthesia, 30-70\% patients reported pain at injection site, ${ }^{[9]} 39 \%$ patients had pain when it was injected at dorsum of hand or wrist, ${ }^{[8]}$ and $92 \%$ patients complained of pain, when no analgesic premedication was given. ${ }^{[10]}$

The commercial preparation of the next decade consisted of an inner phase of pure soybean oil $(100 \mathrm{mg} / \mathrm{ml})$, egg yolk lecithin $(12 \mathrm{mg} / \mathrm{ml})$, and glycerol $(22.5 \mathrm{mg} / \mathrm{ml})$. Soybean oil holds the bulk of propofol in a medium that can be stabilized and dispersed; lecithin serves as the emulsifier to stabilize the propofol-soya oil droplets in aqueous dispersion, and glycerol maintains the formulation isotonic with blood. ${ }^{[7]}$ The boundary of oil layer helps to stabilize the solution by reducing surface tension. The outer aqueous phase comes into contact with intima. Propofol diffuses across the droplet interface and enters the bloodstream. Lower concentration of propofol in aqueous phase of emulsion causes intimal irritation. ${ }^{[11]}$

The impetus for adding microbe growth inhibitors to propofol emulsions occurred in 1990, when clusters of postoperative infections occurred unexpectedly. ${ }^{[3]} \quad$ Ethylenediaminetetraacetate, sodium metabisulfite, tromethamine, benzyl alcohol, benzethonium chloride and sodium benzoate were some of the Patented Propofol Emulsion Excipients. Various formulations containing medium-chain triglycerides, nonemulsion formulations including propofolcyclodextrin and polymeric micelle formulations were also evaluated for the concern of stability of solution, injection pain, hyperlipidemia and postoperative infections. ${ }^{[7]}$

Aquavan GPI (Guilford Pharmaceuticals Inc., Baltimore) 15715, water soluble prodrug was introduced. Chemically a phosphono-O-methyl, it undergoes hydrolysis by alkaline phosphatase liberating propofol as an active metabolite with formaldehyde and phosphate. It has longer half-life, increased volume of distribution, delayed onset and sustained duration of action. Being more potent, it takes longer to achieve peak propofol plasma concentration even with increasing doses and hence is associated with less cardio-respiratory changes. ${ }^{[12,13]}$
Aquafol TM (Daewon Pharmceutical co. 1td. Seol) is a micro emulsion developed to eliminate lipid solvent related adverse events of long-chain triglyceride emulsion Diprivan, (Astra Zeneca, and London) such as infection, fat embolism, hypertriglyceridemia, and pancreatitis. K.M. Kim et al assessed the safety and tolerability of this formulation with respect to its toxicity, skin rash, and pain at injection site, urticaria and increase in total bilirubin content. ${ }^{[14]}$ It was reformulated with $10 \%$ purified Poloxamer 188 (P188) to improve microvascular blood flow by reducing blood viscosity and friction between red blood cells and vessel wall. J.A. Jung studied the induction time, recovery characteristics, pain on injection and postoperative pain due to this formulation in comparison with Diprivan. This micro-emulsion was found to be equally safe and effective (mean infusion rate-6.2 vs $6.3 \mathrm{mg} / \mathrm{kg} /$ hour); it caused more severe injection pain (median VAS $72 \mathrm{~mm}$ vs $11.5 \mathrm{~mm}$ ) which was more frequent $(81.9 \%$ vs $29.2 \%)$ and was reported to be associated with a high aqueous free propofol concentration (63.3 vs $12.4 \mu \mathrm{gm} / \mathrm{ml}$ in Diprivan.) ${ }^{[15]}$

\subsection{Other Causes of propofol pain-}

Immediate pain due to injection of propofol results from its direct irritant effect. Pain originates from afferent nerve endings between intima and media of the veins via myelinated A-delta fibres. Delayed pain is due to kinin cascade; degree of pain depends upon size of vein, volume injected, and duration of exposure of vein wall to propofol. Rapid bolus gets cleared faster and when injected through a larger vein, remains in the midstream of the flow causing minimal intimal irritation. The outer aqueous phase comes into contact with the intima; pain due to injection is reduced by increasing the lipid content and decreasing concentration in the aqueous phase. [16]

\subsection{Pain alleviation methods during administration}

a) At the site IV injection-Propofol should be given preferably through a vein of larger diameter to minimize the contact area of the injection with the intima. ${ }^{[17]}$ Propofol runs through the center of the stream of blood 
thus avoiding the contact with the intima of a larger vein e.g. a forearm vein. Topical nitroglycerine ointment over the proposed site of injection was thought to cause vasodilatation by about $50 \% .{ }^{[18]}$

b) Effect of speed of carrier fluid-The faster speed of carrier fluid dilutes propofol in aqueous solution causing pain. A simple method of stopping the IV fluid while injecting is advocated. ${ }^{[19]}$ Propofol when diluted with venous blood leads to smaller contact area between the drug and the venous endothelium and production of fewer kininogen molecules reduces pain. ${ }^{[20]}$

c) Effect of temperature of injected propofol- Propofol at $4^{\circ} \mathrm{C}$ reduced pain from $46 \%$ to $23 \%$. As observed by Mc Crirrick and Hunter lower temperature reduces the speed of onset of kinin cascade without reducing the efficacy. ${ }^{[21]}$ Fletcher used warm propofol at $37^{\circ} \mathrm{C}$. He explained that high temperature significantly reduced the amount of propofol in aqueous phase by affecting partition co-efficient thus reducing the incidence of pain from $59 \%$ to $22 \%$. $^{[22]}$

d) Premedication mechanisms- The relationship that the injection given more proximally in a larger vein to reduce the percentage of pain therefore leads to a conclusion that by the time, the pain develops the patient is usually asleep. The time lapse between intimal contact and development of kinin cascade has been reported between 5 to 20 seconds. Ephedrine an $\alpha$ and $\beta$ receptor agonist attenuates pain by decreasing release of bradykinin from vascular endothelium; it also releases noradrenaline endogenously, thus producing vasoconstriction. [23, 24] Non-steroidal anti-inflammatory drugs like Ketorolac reduce prostaglandin synthesis; they were given as pretreatment, but failed to reduce the propofol injection pain. ${ }^{[25]}$ Ondansetron has multifaceted actions as sodium channel blocker, 5-HT3 receptor antagonist and a $\mu$-opioid agonist; it may be potentially useful to alleviate pain of propofol in the doses used to induce antiemesis. ${ }^{[26]}$

e) Various induction adjuvants- A well premedicated patient, maintaining airway and saturation and is sedated but arousable is always desirable for induction. The synthetic opioid analgesic pethidine $(1 \mathrm{ml}$, $25 \mathrm{mg}$ ) with propofol induction showed $65 \%$ pain relief, only $2 \%$ patients had significant pain; $21 \%$ patients in placebo group had moderate pain and $23 \%$ had severe pain. Lignocaine the third group for comparison had $57 \%$ pain relief. Local analgesic effect of pethidine is proven and was thought attributable for this. [27] Synergism on co-administration of subhypnotic doses of thiopentone (100mg) and propofol was effective in reducing pain of propofol. $12 \%$ patients of thiopentone group had pain, of which $2 \%$ had severe pain. $88 \%$ pain relief in this group was attributable to the alkalinity and lipid solubility of thiopentone which decreased free concentration of propofol in aqueous phase preventing intimal irritation. [28] Inhibition of bradykinin prevented vasodilatation, hyperpermeability and pain. ${ }^{\text {[29] }}$ Butorphanol (2mg), a strong analgesic with agonist action at kappa and antagonist at opioid mu-receptor, countered propofol pain both centrally \& peripherally. $78 \%$ patients in placebo group and $42 \%$ patients in lignocaine group $(2 \mathrm{ml}, 2 \%)$ had pain. Only $10 \%$ patients of butorphanol group reported pain. ${ }^{[30]}$ Small doses of ketamine $(10 \mu \mathrm{g} / \mathrm{kg}, 50 \mu \mathrm{g} / \mathrm{kg}$ and $100 \mu / \mathrm{kg})$ showed rapid local vascular effects and due to action on peripheral NMDA receptors, reduced pain due to propofol injection. $100 \mu \mathrm{g} / \mathrm{kg}$ dose was optimal as $46.7 \%$ patients reported pain. Three doses were given in three different ways; as premedication $3 \mathrm{~min}$ prior to propofol, just before propofol and mixed with propofol with $66.7 \%, 40 \%$ and $86.7 \%$ reporting of 
pain respectively. ${ }^{[31]}$ Terence Beh et al investigated efficacy of $50 \%$ nitrous oxide in children, to reduce pain during injection of propofol mixed with lignocaine. Treatment group received $50 \%$ nitrous oxide in oxygen during induction with $3 \mathrm{mg} / \mathrm{kg}$ propofol $9.5 \mathrm{ml}$, pre-mixed with lignocaine $1 \% 0.5 \mathrm{ml}$. All patients in both groups were pretreated with EMLA cream on dorsum of hands one hour before surgery to obscure pain of IV cannulation. Pain score in children was judged by a blinded observer as no pain, grimace, grimace + cry and cry + withdrawal as severe pain. Incidence of pain was $4 \%$ after nitrous oxide and $36 \%$ in control group. ${ }^{[32]}$

f) Lignocaine pre-treatment- This has local anesthetic effect on vein wall. Mc Culloch et al suggested the use of $10 \mathrm{mg}$ lignocaine 20 seconds prior to propofol. Pain incidence reduced from $37.5 \%$ in no pre-medication group to $17.5 \%$ in lignocaine group. ${ }^{[33]}$ Ganta and Fee gave 10mg lignocaine 10 seconds prior to propofol and premedicated the patients orally with $10 \mathrm{mg}$ diazepam 90 minutes before operation. Pain of propofol injection reduced from $49.4 \%$ in placebo group to $21.1 \%$ in lignocaine group. ${ }^{\text {[34] }}$

g) Use of lignocaine mixed with propofolHiller and Saarnivaara gave $10 \mathrm{mg}$ lignocaine mixed with propofol and compared it with $10 \mu \mathrm{gm} / \mathrm{kg}$ alfentanil pretreatment; pain incidence was $4 \%$ in lignocaine group to $40 \%$ in alfentanil pretreatment group. ${ }^{[35]}$

A comparison between lignocaine pre-treatment and mixing with propofol was done by Scott. He found that mixing was more effective than pretreatment and reported a significant decrease in the incidence of pain $(46.7 \%$ to $13.5 \%)$ by mixing lignocaine $10 \mathrm{mg}$ with propofol as compared to pretreatment with lignocaine $10 \mathrm{mg} 30$ seconds before propofol injection (from 46.7 to $40 \%$ ). ${ }^{\text {[17] }}$

\section{CONCLUSION}

Propofol has undergone various structural alterations over past few years. Different formulations led to various commercial preparations. Attempts to counter the injection pain, hyperlipidaemia and microbial contamination resulted in utilizing numerous ways and mechanisms to devise an optimal strategy to justify continued use of this revolutionary anaesthetic agent.

\section{REFERENCES}

1. RD Miller, LI Eriksson, JP Wiener-Kronish, W1 Young. Intravenous Anaesthetics, In: Miller's Anaesthesia. $7^{\text {th }}$ ed, Ch.26. Philadelphia USA: Elsevier 2010 p. 719-768

2. L.Wang, B.Wu, and Y. Sun: "Translocation of protein kinase $\mathrm{C}$ isoforms is involved in propofol induced endothelial nitric oxide synthase activation." British Journal of Anaesthesia, 2010; 104: 606-612.

3. Bennett SN, McNeil MM, and Bland LA: "Postoperative infections traced to contamination of an intravenous anaestheticpropofol."New England Journal of Medicine, 1995; 333: 147-54.

4. Laxenaire MC, Mata-Bermejo E: "Lifethreatening anaphylactoid reactions to propofol.” Anaesthesiology, 1992; 77: 27580 .

5. de Leon-Casasola OA, Weiss A: "Anaphylaxis due to propofol." Anaesthesiology, 1992; 77: 384-86.

6. P.C.A. Kam, D.Cardone: "Propofol infusion syndrome." Review Article. Anaesthesia, 2007; 62: 690-701.

7. Baker MT, Naguib M: "Propofol: The Challenges of Formulation." Anaesthesiology, 2005; 103: 860-876.

8. L. P. Briggs, R. S. Clarke, J. W. Dundee, J. Moore, M. Bahar, P. J. Wright: "Use of Diisopropyl phenol as a main agent for short 
procedures." British Journal of Anaesthesia, $1981 ; 53: 1197-1202$.

9. Doenicke. A, Nebauer. A. E, Hoernecke. R:“Osmolalities of propylene glycol containing drug formulations for parenteral use. Should propylene glycol be used as a solvent?"Anaesthesia\& Analgesia, 1992; 75:431-435.

10. Yoshitaka Fuji, MichiyoItakura: "Comparison of Lidocaine, Metoclopramide and Flurbiprofenaxetil to prevent pain due to Propofol injection." Clinical therapeutics, 2008; 30; 2:280-286.

11. A.W.Doenicke,M.F.Roizen,W.Kellermann, J.Babl:"Reducing pain due to Propofol injection,the role of solvent."Anaesthesia\& Analgesia,1996; 82:472-474.

12. Gibiansky E, Struys Michel M. R. F: "AQUAQVAN Injection, a Water-soluble prodrug of Propofol, as a bolus Injection: A Phase I Dose-escalation Comparison with Diprivan"- Part 1 Anaesthesiology, 2005; 103: 718-729.

13. Struys Michel M. R. F, Gibiansky Ekaterina: "AQUAQVAN Injection, a Water-soluble prodrug of Propofol, as a bolus Injection: A Phase I Dose-escalation Comparison with Diprivan"- Part 2. Anaesthesiology, 2005; 103:730-743.

14. Kim KM, Choi BH: Pharmacokinetics and "Pharmacodynamics of Propofol Microemulsion and Lipid Emulsion after an Intravenous Bolus and Variable Rate Infusion."Anaesthesiology, 2007; 106: 924-934.

15. J. A. Jung, B. M. Choi, S. H. Cho: "Effectiveness, Safety, and Pharmacokinetic and Pharmacodynamic Characteristics of MicroemulsionPropofol in Patients Undergoing Elective Surgery under Total Intravenous Anaesthesia." British Journal of Anaesthesia, 2010; 104: 563-76.
16. C.H.Tan and M.K.Onsiong: "Pain on injection of propofol." Review Article. Anaesthesia, 1998; 53:468-476.

17. Scott R.P.F, Saunders, Norman J: "Propofol: Clinical strategies for preventing pain on injection." Anaesthesia, 1988; 43:492-494.

18. Jerome.F,O'Hara,Juraj Sprung: "Effects of topical Nitro glycerine and intravenous Lignocaine on Propofol-induced pain on injection." Anaesthesia Analgesia, 1997; 84:865-869.

19. C.L Huang, Y.P Wang, YJ Cheng: "The effect of carrier intravenous fluid speed on the injection pain of Propofol." Anaesthesia \& Analgesia, 1995; 81:1087-1088.

20. D.S.McDonald, P.Jameson: "Injection pain with Propofol: Reduction with aspiration of blood. "Anaesthesia, 1996; 51:878-880.

21. A.McCrirrick, S.Hunter: "Pain on injection of Propofol: The effect of injectate temperature." Anaesthesia, 1990; 45:443444.

22. Fletcher GC, Gillespie JA, Davidson JA: "The effect of temperature upon pain during injection of Propofol." Anaesthesia, 1996; 51: 498-99.

23. Mi. A. Cheong, Kyo S. Kim, Won. J Choi: "Ephedrine reduces the pain from Propofol injection." Anaesthesia \& Analgesia, 2002; 95:1293-1296.

24. F.Gamlin, M.Vucevic, L.Winslow, J.Berridge: "The hemodynamic effects of Propofol in combination with Ephdrine." Anaesthesia, 1996; 51:488-491.

25. A.J.Smith, W.I.Power: "The effect of pretreatment with Ketorolac on pain during intravenous injection of Propofol." Anaesthesia, 1996; 51:883-885.

26. S.P.Ambesh, P.K.Dubey, P.K.Sinha: "Ondansetron pre-treatment to alleviate pain on Propofol injection.” Anaesthesia \& Analgesia, 1999; 89:197-199. 
27. B.Lyons, D. Lohan, C. Flynn: "Modification of pain on injection of Propofol: A comparison of Pethidine and Lignocaine." Anaesthesia, 1996; 51: 394-95.

28. T.W.Lee, A.W.Loewenthal, Strachan J.A, Todd B.D: "Pain during injection of Propofol: The effect of prior administration of Thiopentone." Anaesthesia, 1994;49:817-818.

29. A.Agrawal, M.Ansari, and D. Gupta: "Pretreatment with Thiopental for prevention of Pain associated with Propofol Injection." Anaesthesia \&Analgesia, 2004;98:683-86.

30. A.Agarwal, Mehdi Raja, and Sanjay Dhiraaj: "Pain during injection of Propofol: The effect of prior administration of Butorphanol." Anaesthesia \& Analgesia, 2004; 99:117-119.

31. SeungWoo Koo, Sun-Jun Cho, Young-Kug Kim. "Small dose of Ketamine reduces Pain of Propofol injection." Anaesthesia \& Analgesia, 2006; 103:1444-47.

32. Terence Beh, William Splinter: "In children, nitrous oxide decreases pain on injection of propofol mixed with lidocaine." Canadian Journal of Anaesthesia, 2002; 49: 1061-63.

33. Mc Culloch MJ, LeesN: "Assessment and modification of pain on induction with Propofol." Anaesthesia, 1985; 40: 1117-20.

34. Ganta R, Fee JP: "Pain on injection of Propofol: comparison of Lignocaine with Metoclopramide." British Journal of Anaesthesia, 1992; 69: 316-17.

35. Hiller A, Saarnivaara L: "Injection pain, cardiovascular changes and recovery following induction of anaesthesia with Propofol in combination with alfentanil or lignocaine in children." Acta Anaesthsiologica Scandinavica., 1992; 36: 564-68. 